\title{
An in-situ MRI method for quantifying temperature change of hydrate growth in a porous medium
}

\author{
Lunxiang Zhang ${ }^{1}$, Mingrui Sun ${ }^{1}$, Lei Yang ${ }^{1}$, Xiaotong Zhang ${ }^{2}$, Jiafei Zhao ${ }^{1}$, and Yongchen \\ Song ${ }^{1}$
}

${ }^{1}$ Dalian University of Technology

${ }^{2}$ Zhejiang University

March 14, 2021

\begin{abstract}
The intricacy of the thermo-hydro-chemically coupled process of hydrate phase transition requires real-time in-situ observations, therefore, thermometry maps are of particular value to reveal the heat transfer process during crystal growth and dissociation. By using the temperature dependence of water proton chemical shift, the temporally- and spatially-resolved thermometry of tetrahydrofuran hydrate growths is presented in this study. Images of temperature changes were synchronously obtained by a 9.4 T 1H Magnetic Resonance Imaging (MRI) system, in order to predict the saturation of aqueous solution, solid hydrate phases, and the positive temperature anomaly of exothermic reaction. Variations of MRI signal decrease and histories of temperature rise differ significantly in space and time, which have a great use for analyzing the physical micro-mechanism and the heat transfer process of hydrate growth. The extension of these predicted results could have important implications for optimizing the phase transition process of gas hydrates.
\end{abstract}

\section{Hosted file}

manuscript.pdf available at https://authorea.com/users/401415/articles/513489-an-in-situmri-method-for-quantifying-temperature-change-of-hydrate-growth-in-a-porous-medium 